\title{
Cooperative autoinhibition and multi-level activation mechanisms of calcineurin
}

\author{
Sheng-Jie Li ${ }^{1, *}$, Jue Wang ${ }^{1,{ }^{*}}$, Lei Ma ${ }^{2,{ }^{*}}$, Chang Lu ${ }^{1}$, Jie Wang ${ }^{1}$, Jia-Wei Wu ${ }^{1,3}$, Zhi-Xin Wang ${ }^{1,2}$ \\ ${ }^{1}$ MOE Key Laboratory of Protein Science, School of Life Sciences, Tsinghua University, Beijing 100084, China; ${ }^{2}$ Institute of Bio- \\ physics, Chinese Academy of Sciences, Beijing 100101, China; ${ }^{3}$ Tsinghua-Peking Center for Life Sciences, Tsinghua University, \\ Beijing 100084, China
}

The $\mathrm{Ca}^{2+}$ /calmodulin-dependent protein phosphatase calcineurin $(\mathrm{CN})$, a heterodimer composed of a catalytic subunit $A$ and an essential regulatory subunit $B$, plays critical functions in various cellular processes such as cardiac hypertrophy and $\mathrm{T}$ cell activation. It is the target of the most widely used immunosuppressants for transplantation, tacrolimus (FK506) and cyclosporin A. However, the structure of a large part of the CNA regulatory region remains to be determined, and there has been considerable debate concerning the regulation of $\mathrm{CN}$ activity. Here, we report the crystal structure of full-length $\mathrm{CN}$ ( $\beta$ isoform), which revealed a novel autoinhibitory segment (AIS) in addition to the well-known autoinhibitory domain (AID). The AIS nestles in a hydrophobic intersubunit groove, which overlaps the recognition site for substrates and immunosuppressant-immunophilin complexes. Indeed, disruption of this AIS interaction results in partial stimulation of $\mathrm{CN}$ activity. More importantly, our biochemical studies demonstrate that calmodulin does not remove AID from the active site, but only regulates the orientation of AID with respect to the catalytic core, causing incomplete activation of $\mathrm{CN}$. Our findings challenge the current model for $\mathrm{CN}$ activation, and provide a better understanding of molecular mechanisms of $\mathrm{CN}$ activity regulation.

Keywords: structure of full-length calcineurin; bipartite autoinhibition; multi-level activation; mechanism of immunosuppressant stimulation; regulation of calcineurin

Cell Research (2016) 26:336-349. doi:10.1038/cr.2016.14; published online 22 January 2016

\section{Introduction}

Calcineurin $(\mathrm{CN})$, also known as protein phosphatase 2B (PP2B), is the only known protein Ser/Thr phosphatase directly regulated by calcium and calmodulin $(\mathrm{CaM})$ [1-3]. Coupling $\mathrm{Ca}^{2+}$ signals to cellular responses, $\mathrm{CN}$ has diverse biological functions and plays a critical role in many physiological processes, including the immune response, apoptosis, muscle differentiation, bone formation, and neuronal signaling. Among the best-characterized substrates of $\mathrm{CN}$ are the nuclear factor of activated

\footnotetext{
*These three authors contributed equally to this work.

Correspondence: Zhi-Xin Wang ${ }^{\mathrm{a}}$, Jia-Wei Wu

${ }^{\mathrm{a}}$ Tel: +86-10-6278-5505; Fax: +86-10-6279-2826

E-mail: zhixinwang@mail.tsinghua.edu.cn

${ }^{\mathrm{b}}$ Tel: +86-10-6278-9387; Fax: +86-10-6279-2826

E-mail: jiaweiwu@mail.tsinghua.edu.cn

Received 20 July 2015; revised 12 October 2015; accepted 27 November 2015; published online 22 January 2016
}

T cells (NFAT) family of transcription factors [4-6]. CN directly binds to NFAT transcription factors in the cytoplasm, resulting in their dephosphorylation and subsequent translocation into the nucleus. Due to its role in $\mathrm{T}$ cell activation, $\mathrm{CN}$ has been intensively targeted for the development of immunosuppressant drugs. The two most successful inhibitors discovered so far are cyclosporin A (CsA) and tacrolimus (FK506) [7-11], which bind to intracellular proteins cyclophilin A (CyPA) and FKBP12, respectively. The resultant CyPA-CsA and FKBP12FK506 complexes inhibit dephosphorylation of NFAT proteins and hence block their nuclear import.

$\mathrm{CN}$ is a heterodimeric phosphatase consisting of a catalytic subunit calcineurin A (CNA) and a regulatory subunit calcineurin $\mathrm{B}$ (CNB; Figure $1 \mathrm{~A})$. In addition to the catalytic domain, CNA contains a B-subunit-binding helix (BBH), a CaM-binding domain (CBD) and an autoinhibitory domain (AID), which together comprise the regulatory domain $(\mathrm{RD})$ of the A subunit. The regulatory subunit CNB is a member of the "EF-hand" protein 
A

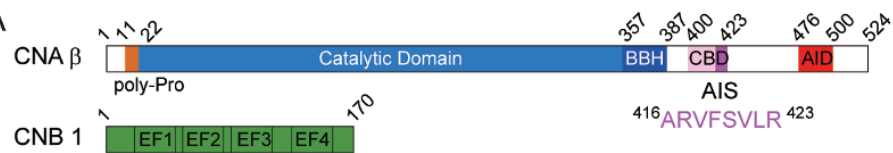

B mouse $\mathrm{CN} \alpha$

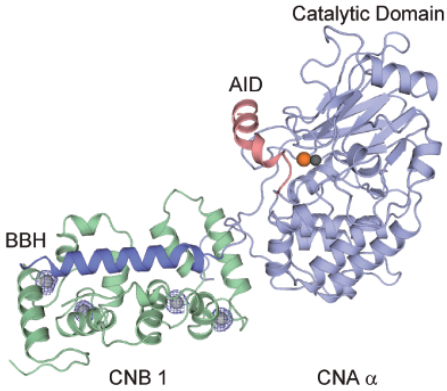

C human $\mathrm{CN} \beta$



$E$
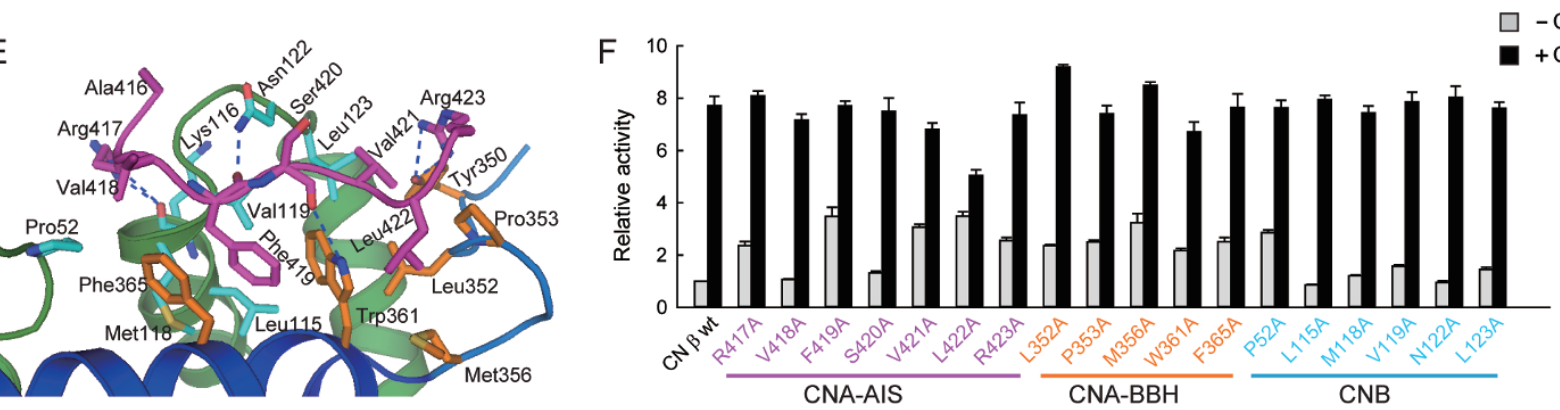

Figure 1 Comparison of $\mathrm{CN} \alpha$ and $\beta$ structures. (A) Domain organization of $\mathrm{CN} \beta$. The key structural elements are indicated. (B) Ribbon representation of mouse $\mathrm{CN} \alpha$ structure. (C) Ribbon representation of human $\mathrm{CN} \beta$ structure. The inset shows the SA-omit map (contoured at $2.0 \sigma$ ) for AIS. The SA omit maps for the calcium ions in both $\mathrm{CN} \alpha$ and $\mathrm{CN} \beta$ structures are shown in $\mathbf{B}$ and $\mathbf{C}$, contoured at $2.0 \sigma$. (D) Superposition of $\mathrm{CN} \alpha$ and $\beta$ isoforms. (E) Interactions between AIS and the intersubunit groove. Key residues are highlighted as sticks and colored as follows: AIS (magenta), BBH (orange), CNB (cyan). Hydrogen bonds are shown as dotted lines. (F) Activities of WT and mutant CN $\beta$ proteins towards $p$ NPP in the absence or presence of CaM. All assays were performed in the presence of $1 \mathrm{mM} \mathrm{Ca}^{2+}$ and $1 \mathrm{mM} \mathrm{Mn}^{2+}$. The assays were performed in triplicate, and data shown represent the Mean \pm SEM from three experiments.

family. It contains four $\mathrm{Ca}^{2+}$-binding loops and is tightly bound to CNA-BBH. A widely accepted model for $\mathrm{CN}$ activation involves sequential changes in $\mathrm{CN}$ conformation $[3,12]$. At low $\mathrm{Ca}^{2+}$ concentrations, $\mathrm{CN}$ exists in an inactive state in which only two high-affinity binding sites on $\mathrm{CNB}$ are occupied by $\mathrm{Ca}^{2+}$. Meanwhile, in CNA, CBD interacts with $\mathrm{BBH}$, and AID acts as a pseudosubstrate and blocks the catalytic site (Form I or the resting conformation). In response to elevated calcium levels, the occupancy of the low-affinity sites on CNB by $\mathrm{Ca}^{2+}$ causes dissociation of $\mathrm{CBD}$ from $\mathrm{BBH}$, resulting in the $\mathrm{CN}$ transition from the resting state to a partially active state (Form II). The subsequent binding of CaM leads to displacement of AID from the active site and full $\mathrm{CN}$ activation (Form III).

While this CaM-dependent $\mathrm{CN}$ activation model ex- plains the biochemical and biophysical results, several fundamental questions remain to be addressed. There is limited information on how the resting $\mathrm{CN}$ undergoes conformational changes which cause the $\mathrm{CN}$ transition from Form I to Form II upon $\mathrm{Ca}^{2+}$ binding to the low-affinity sites of CNB [13]. In the presence of excess $\mathrm{Ca}^{2+}$, kinetic studies using $\mathrm{CN}$ truncation mutants indicated the presence of additional autoinhibitory elements within the RD of CNA in calcium-loaded CN (Form II) [14, 15]. However, the precise sequence of the autoinhibitory element and its autoinhibitory mechanism are still unclear. Almost all CN structures currently available are either in the ligand-bound states or in the truncated forms, in which most of the RD sequence is absent from the expression constructs. The structure of human $\mathrm{CN} \alpha$ (PDB ID code: 1AUI) is the only apo one that contains 
an intact RD sequence [9]. In this structure, a major part of $\mathrm{RD}$ is disordered and missing in the structure, with only a short, ordered AID helix observed near the active site. Therefore, how the other autoinhibitory elements in CNA-RD exert their autoinhibitory effects remains elusive. Recently, Ye et al. [16] reported a crystal structure of $\mathrm{CN}$ covalently linked to CaM (PDB ID code: 4IL1), which retains full CaM-activated activity. Surprisingly, the crystal structure showed virtually no structural change in the active site and no evidence of CaM despite being covalently linked. Therefore, more structural information is required to fully elucidate the molecular basis of $\mathrm{CN}$ activation.

Here, we describe the second high-resolution structure of full-length $C N$ ( $\beta$ isoform), which reveals a novel autoinhibitory segment (AIS) within the RD of CNA. This segment is located at the C-terminal boundary of CBD in $\mathrm{CN}$ and packs into the hydrophobic groove that was previously identified as the binding site of immunosuppressants and substrates (the LxVP-binding site). Our structural and biochemical analyses reveal that RD of CNA employs a bipartite binding mode involving interactions of both AID and AIS with the CN catalytic core, and that $\mathrm{CaM}$ binding is not sufficient to completely relieve the cis-autoinhibitory effect of AID on the active site of $\mathrm{CN}$. By combining these results with previously published data, we propose a new model, in which $\mathrm{CN}$ is regulated by a set of intramolecular interactions and multi-level activation mechanisms.

\section{Results}

\section{Crystal structures of mouse $C N \alpha$ and human $C N \beta$}

There are three human isoforms of CNA $(\alpha, \beta$ and $\gamma)$ and two isoforms of CNB (1 and 2) (Supplementary information, Figure S1). The two main isoforms of CNA ( $\mathrm{A} \alpha$ and $\mathrm{A}$ ) bind to a common regulatory $\mathrm{B} 1$ subunit, whereas the $\gamma$ isoform interacts only with a testis-specific regulatory $\mathrm{B} 2$ subunit. To elucidate the molecular basis of $\mathrm{CN}$ auto-regulation, we determined a $3.1 \AA$ structure of mouse $\mathrm{CN} \alpha(\mathrm{CNA} \alpha+\mathrm{CNB} 1)$ and two structures of human $\mathrm{CN} \beta$ (wild-type (WT) or mutant $\mathrm{CNA} \beta+\mathrm{CNB} 1$ ) (Supplementary information, Table S1). Although mouse and human $\mathrm{CN} \alpha$ sequences differ by a 10 -amino-acid insertion in the $\mathrm{C}$-terminal domain of human $\mathrm{CNA} \alpha$, our structure of mouse $\mathrm{CN} \alpha$ is nearly identical to that of human $\mathrm{CN} \alpha$ revealed by a previous report [9] and most of the C-terminal region of CNA apart from the AID is missing in both structures (Figure 1B and Supplementary information, Figure S2A). The full-length human $\mathrm{CN} \beta$ was crystallized in the hexagonal space group $\mathrm{P}_{5} 22$, and the crystals diffracted to a resolution of $2.2 \AA$ (Figure 1C and Supplementary information, Table S1). The atomic model includes a CNB fragment (except for three most N-terminal residues) and a CNA fragment that includes the catalytic domain, BBH, part of CBD, and AID. Four calcium ions are individually coordinated by each $\mathrm{EF}$ hand of CNB. AID (residues 478-495) folds into an $\alpha$-helix that lies over the substrate-binding cleft in the catalytic domain. In the human $\mathrm{CN} \beta$ structure, we observed a region of contiguous positive difference electron density $\left(\mathrm{F}_{\text {obs }}-\mathrm{F}_{\text {calc }}\right)$ in the interface of CNA and CNB of $\sim 21 \AA$ in length. The strong side chain feature was assigned as the aromatic residue Phe419 and the remaining residues were then manually built (Figure 1C, inset and Supplementary information, Figure S2C). The amino acid sequence of this region was assigned as ${ }^{416}$ ARVFSVLR $^{423}$. The absence of electron density for residues 388-415, 424-477 and 496-524 suggests that these regions remain flexible. To further confirm this observation, we also crystallized a human $\mathrm{CN} \beta$ mutant $\left(\mathrm{CN}_{\mathrm{V} 418 \mathrm{Y} / \mathrm{F} 419 \mathrm{~L}}\right)$ and determined its structure (Supplementary information, Table S1). The structure of mutant $\mathrm{CN} \beta$ is almost identical to that of WT CN $\beta$ (r.m.s.d of $0.2 \AA$ over $482 \mathrm{C} \alpha$ atoms), and the mutated sequence of ${ }^{416}$ ARYLSVL ${ }^{422}$ can be built into the electron density as well (Supplementary information, Figure S2B and S2D). Interestingly, the corresponding sequence has not been seen in the apo structures of fulllength human (PDB ID code: 1AUI) and mouse $\mathrm{CN} \alpha$ (Supplementary information, Figure S2A), suggesting that either the location of this region is specific for $\mathrm{CN} \beta$ or this region is as flexible as other invisible residues in the $\mathrm{CN} \alpha$ structures.

Topologically, the structure of $\mathrm{CN} \beta$ is similar to that of $\mathrm{CN} \alpha$. Superimposition of the CNB subunits in the $\mathrm{CN} \alpha$ and $\beta$ structures revealed a relative rotation of the CNA subunits by $5^{\circ}$ (Figure 1D). In contrast to the $\alpha$ and $\gamma$ isoforms of CNA, the N-terminus of the $\beta$ isoform contains a poly-Pro motif that may contribute to differential substrate specificities of the isoforms [17]. In the $\mathrm{CN} \beta$ structure, this proline-rich segment is largely ordered, but has no direct contacts with the active site or the substrate-binding region (Figure $1 \mathrm{C}$ ). The most important feature of our $\mathrm{CN} \beta$ structure is that the $\mathrm{C}$-terminal 8 residues of CBD $\left({ }^{416} \mathrm{ARVFSVLR}{ }^{423}\right.$, here termed AIS for autoinhibitory segment, see below) interact with a hydrophobic groove formed at the junction of CNA and CNB subunits (Figure 1E), resulting in the burial of $\sim 1$ $100 \AA^{2}$ of solvent-accessible surface area. The predominantly hydrophobic interactions recruiting AIS mainly involve residues from the $\mathrm{N}$-terminal region of $\mathrm{BBH}$ and the preceding loop in CNA (CNA residues 352-365), and the C-terminal helix of the third EF-hand motif and the following linker region in CNB (CNB residues 115-123). 
In particular, Phe419 of AIS penetrates into a hydrophobic pocket formed by Trp361 and Phe365 from CNA and Leu115, Met118 and Val119 from CNB. The highly conserved Leu422 nestles into an adjacent pocket lined by CNA residues Leu352, Pro353, Met356 and Trp361. In addition, Val421 between these two essential amino acids is in contact with Trp361 from CNA and Leu123 from $\mathrm{CNB}$, and the flanking residues Ala416, Arg417 and Val418 also make weak van der Waals interactions with Phe365 of CNA and Pro52 and Met118 of CNB. Additional polar interactions are formed between two mainchain carbonyl oxygens of AIS and Trp361 (CNA) and Asn122 (CNB) from the intersubunit groove. Therefore, hydrophobic interactions likely provide the driving force for the recruitment of AIS.

To assess the importance of AIS, we generated a series of point mutations in human $\mathrm{CN} \beta$ heterodimer and examined their effects on $\mathrm{CN}$ catalytic activity using $p$ NPP ( $p$-nitrophenyl phosphate) as the substrate with an excess amount of $\mathrm{Ca}^{2+}$ (Figure 1F). $p$ NPP is a non-proteinaceous, non-specific substrate used to assay alkaline and acid phosphatases, and tyrosine and serine/threonine phosphatases such as PP1, PP2A, PP2B $(\mathrm{CN})$ and PP2C. The phosphatase activity of WT or mutant $\mathrm{CN} \beta$ towards $p$ NPP was measured using a continuous or single-point spectrophotometric assay based on the ability of phosphatases to catalyze the hydrolysis of $p$ NPP to $p$-nitrophenol, a chromogenic product with absorbance at $410 \mathrm{~nm}$. The small $p$ NPP molecule binds directly to the enzyme active site and therefore can be used to probe the properties of the enzyme active site $[15,18]$. When residues in AIS were individually replaced by alanine, the catalytic efficiencies of most mutants (R417A, F419A, V421A, L422A and R423A) were increased by 3-4-fold in the absence of CaM, whereas the V418A and S420A mutations had little effect on CN activity. Replacement of the interacting hydrophobic residues in CNA-BBH by alanine yielded a modest increase in CaM-independent activities. The increase of activities of the interface mutants indicated that binding of AIS to the intersubunit groove may play a role in $\mathrm{CN}$ autoinhibition. All mutant proteins retain the CaM-binding capacities (Supplementary information, Figure S3) and can be further activated by CaM (Figure 1F). Although AIS is part of the CBD, the AIS mutations do not seem to affect CaM binding to $\mathrm{CBD}$, probably because CaM strongly interacts with CBD with a $K_{\mathrm{d}}$ in the low picomolar range [19] and AIS is not critical for the CBD-CaM interaction.

The CNA-CNB interface is a general recognition site for AIS, inhibitors and substrates

The substrates and regulators of $\mathrm{CN}$ contain at least one of the two conserved CN-binding motifs, PxIxIT and LxVP, which interact with two distinct docking sites on $\mathrm{CN}$ [3, 20-23]. Recently, Grigoriu et al. [24] reported the first high-resolution structure of $\mathrm{CN} \alpha$ bound to a physiological binding partner, the protein inhibitor A238L from African swine fever virus. The structure of the $\mathrm{CN}-$ A238L complex reveals that the interface between these two proteins consists of two discontinuous contact regions. In addition to the interaction of residues 206-211 in A238L with the PxIxIT-binding site in CNA, residues ${ }^{229} \mathrm{LCVK}^{232}$ in $\mathrm{A} 238 \mathrm{~L}$, which are analogous to the LxVP motif, interact with a CNA-CNB interface region that sits over $25 \AA$ away from the active site. Trp352 and Phe356 from CNA $\alpha$ appear to be the most important residues for CN-LxVP interaction. These residues correspond to Trp361 and Phe365 in human CNA $\beta$ (Figure $2 \mathrm{~A})$. Comparison between the structures of $\mathrm{CN} \beta$ and the $\mathrm{CN}-\mathrm{A} 238 \mathrm{~L}$ complex reveals that the interaction of AIS $\left({ }^{419} \mathrm{FSVL}^{422}\right)$ with the CNA-CNB interface is very similar to the CN-LxVP interaction observed in the CN-A238L complex (Figure 2B and 2C). The structure of the $\mathrm{CN}$ V418Y/F419L mutant of $\mathrm{CN} \beta$ provides further support for this observation (Figure 2D). Indeed, Leu419 in the mutant appears to interact in the same way as Leu229 in the CNA238L complex. Notably, Tyr418 in $\mathrm{CN}_{\mathrm{V} 418 \mathrm{Y} / \mathrm{F} 419 \mathrm{~L}}$, which is immediately $\mathrm{N}$-terminal to the LxVP motif, fits into a deep pocket formed by the loops connecting EF hands 1 and 2 and EF hands 3 and 4 of CNB. This is consistent with the experimental observations that many LxVP motifs are immediately preceded by an aromatic residue (Phe or Tyr) [23], which may act as a binding strength enhancer.

Although the structure of $\mathrm{CN}$ bound to a bona fide substrate has yet to be determined, the structure of the $\mathrm{CN}-\mathrm{A} 238 \mathrm{~L}$ complex provides the structural basis for the computational model of $\mathrm{CN}$ with a phosphosubstrate bound to its active site [24]. The $p$ RII peptide (a 19-amino-acid phosphopeptide) contains a typical LxVP motif that is required for its recognition and efficient dephosphorylation by CN. Grigoriu et al. [24] used bias-exchange metadynamics MD to generate the first computational model of $\mathrm{CN}$ in complex with the RII phosphopeptide. In this model, LDVP of $p$ RII interacts with the LxVP-binding pocket and phospho-Ser95 interacts with the $\mathrm{CN}$ active site. We therefore examined the dephosphorylation of $p$ RII peptide by the alanine-substitution $\mathrm{CN} \beta$ mutants (Figure 2E). As expected, impairing AIS interaction resulted in increased CaM-independent activity towards the $p$ RII peptide, similar to the $p$ NPP assays. All AIS mutants can be fully activated by CaM, as the LxVP-binding sites in these mutants are intact. By contrast, the $\mathrm{BBH}$ substitutions that were designed to 
A

hCNA $\propto 326$ NNVMNIRQFNCSPHPYWLPNF DVFTWSLP VGEKVTEMLVNVLNICSDD

hCNA $\beta 335$ NNVMNIRQFNCSPHPYWLPNF DVFTWSLP VGEKVTEMLVNVLS ICSDD

\section{CBBD}

hCNA $\alpha 376$ ELGSE-EDGFDGATAAARKEVIRNKIRAIGKMARVFSVLREESESVITLK hCNA $\beta 385$ ELMTEGEDQFDGS-AAARKEIIRNKIRAIGKMARVFSVLREESESVLTLK

B $\mathrm{CN} \alpha$ in complex with $\mathrm{A} 238 \mathrm{~L}$

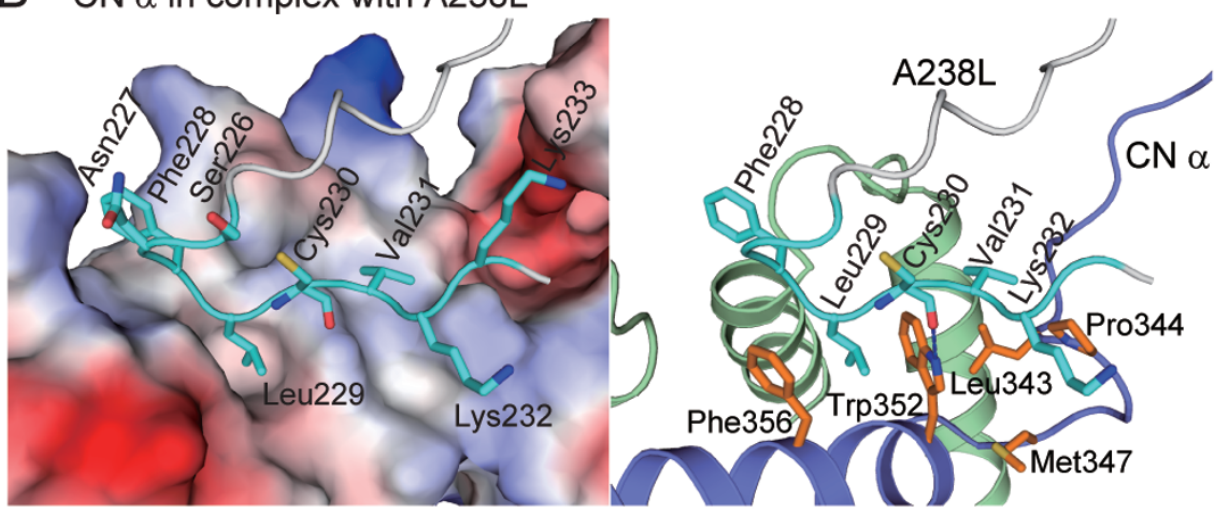

C $\mathrm{CN} \beta$

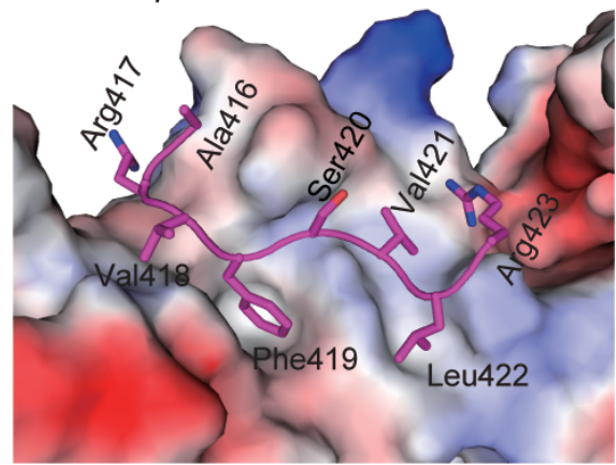

D $\mathrm{CN}_{\mathrm{V} 418 \mathrm{Y} / \mathrm{F} 419 \mathrm{~L}}$

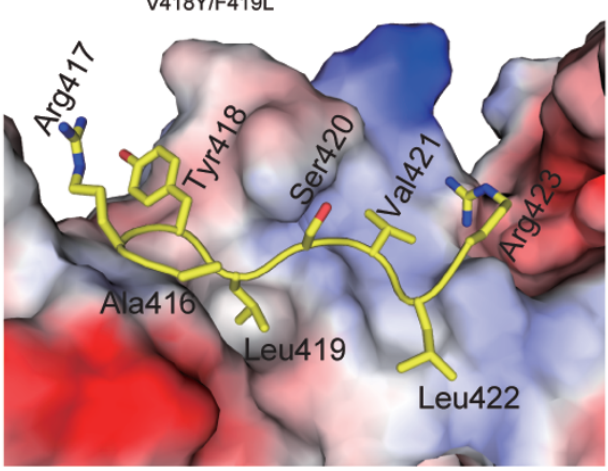

$E$

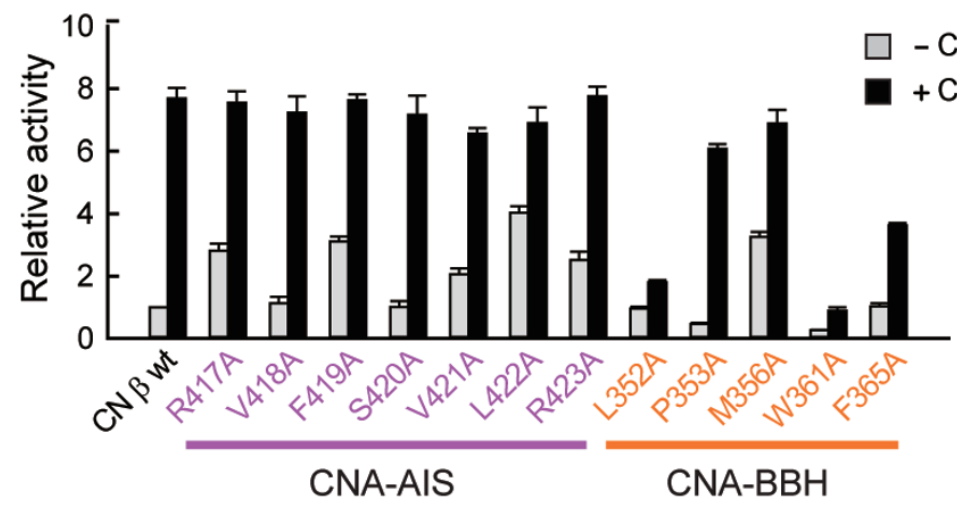

Figure 2 The autoinhibition mechanism of AIS. (A) Sequence alignment of the regulatory region from CNA $\alpha$ and $\beta$ isoforms. (B) Interactions between $\mathrm{CN} \alpha$ and A238L (PDB ID code: 4F0Z). The color scheme of $\mathrm{CN} \alpha$ is the same as in Figure 1B, and the inhibitor A238L is shown in grey with key residues highlighted in cyan. $\mathrm{CN} \alpha$ in the left panel is shown in electrostatic representation. (C, D) Surface representations of the AIS-binding grooves on CN $\beta$ structures. Phe419 in WT CN $\beta$ (C) and Leu419 in mutant $C N \beta$ (D) penetrate into the same hydrophobic pocket. (E) Relative activities of CN $\beta$ mutants towards the $p$ RII peptide in the absence or presence of CaM. (Mean \pm SEM; $n=3$ ). All assays were performed in the presence of $1 \mathrm{mM} \mathrm{Ca}^{2+}$ and $1 \mathrm{mM} \mathrm{Mn}^{2+}$. 
impair the LxVP recognition displayed dramatic impact on $p$ RII dephosphorylation. Particularly, the activities of mutants L352A, W361A and F365A were significantly reduced even in the presence of $\mathrm{CaM}$, as these residues participate in the recognition of the LxVP motif in $p$ RII substrate (Figure 2E). Inspection of the docked $p$ RII peptide on $\mathrm{CN} \alpha$ shows that the $\mathrm{C}$-terminal portion of the peptide collides with AID of CNA $\alpha$. The model of the $\mathrm{CN}-p \mathrm{RII}$ complex and the mutagenesis results suggest that the autoinhibitory peptide, AID or AIS, inhibits $\mathrm{CN}$ either by targeting its active site or by occupying a critical substrate-binding region of $\mathrm{CN}$ (distant from the active site).

AIS and AID cooperatively inhibit enzyme activity of $C N$

We next examined the effects of step-wise C-terminal truncations of the catalytic subunit A on enzyme activity in the presence of excess $\mathrm{Ca}^{2+}$ (Figure 3A). Most truncation mutants were generated by including an internal thrombin-recognition site in human or mouse full-length $\mathrm{CN}$ (Supplementary information, Data S1). All truncation mutants of $\mathrm{CNA} \beta$, except for $\mathrm{CNA}_{1-356}$, can bind to $\mathrm{CNB}$, indicating that these truncations did not affect the CNA-CNB interaction (Supplementary information, Figure S4A). Phosphatase activities of WT and mutant CNs were measured using $p$ NPP or $p$ RII as the substrate in the absence or presence of CaM. As shown in Figure $3 \mathrm{~B}$, in the absence of CaM, WT CN displayed a low basal activity towards both $p$ NPP and $p$ RII, and binding of $\mathrm{CaM}$ to $\mathrm{CN}$ resulted in $\sim 10$-fold activation. Removal of the A subunit $\mathrm{C}$-terminal region by truncation at residue 450 generated a mutant $\left(\mathrm{CN}_{1-450}, \mathrm{M} 1\right)$ that is fully activated towards $p$ NPP but still requires CaM for full activity towards $p$ RII. Truncation of the A subunit at residue 414 $\left(\mathrm{CN}_{1-414}, \mathrm{M} 2\right)$ resulted in completely CaM-independent phosphatase activity towards both substrates. These results provide clear evidence for the presence of additional autoinhibitory elements within residues 415-450 of CNA that can be relieved by CaM binding. Further removal of the CBD region located within residues 398-414 by truncation at residue $397\left(\mathrm{CN}_{1-397}, \mathrm{M} 3\right)$ also gave a fully active, CaM-independent form. The catalytic domain of $\mathrm{CN}\left(\mathrm{CNA}_{1-356}, \mathrm{M} 4\right)$ alone had the lowest phosphatase activity for $p$ RII compared with the BBH-containing mutants, indicating that $\mathrm{CNA}$ and $\mathrm{CNB}$ are both required for the dephosphorylation of this peptide substrate. Sequence alignment shows that the newly identified regulatory segment AIS is highly conserved in the three CNA isoforms (Figure 2A and Supplementary information, Figure S1). We therefore carried out biochemical and mutagenesis studies of mouse $\mathrm{CN} \alpha$. Similar to $\mathrm{CN} \beta$, removal of AID (residues 457-481) did not result in complete activation of $\mathrm{CN}$ (towards $p \mathrm{RII}$ ) and fully CaM-independent activity required an additional removal of residues 405-441 (Figure 3C). These results indicate that although the AIS residues are untraceable in the crystal structure of $\mathrm{CN}$ $\alpha$ due to their flexibility, the bipartite autoinhibition is a common feature of $\mathrm{CN}$ isoforms.

In order to further investigate the contribution of the C-terminal region of CNA to $\mathrm{CN}$ activity, we conducted phosphatase assays using the CaM-independent form of human $\mathrm{CN}$, hCN $\beta_{1-397}$, in the presence of various C-terminal segments of CNA (Figure 3D). $\mathrm{CN}_{1-397}$ contains the intact BBH but lacks CBD and AID. As shown in Figure $3 \mathrm{D}$, all three fragments can completely trans-inhibit the phosphatase activity of $\mathrm{CN}_{1-397}$, even though they may bind to different sites in $\mathrm{CN}_{1-397}$. The AID peptide was a moderate inhibitor of $\mathrm{CN}_{1-397}$, whereas the $\mathrm{CBD}$ peptide was a much weaker inhibitor. A 102-amino-acid fragment of CNA containing both AID and CBD (residues 398$500, \mathrm{RD}_{398-500}$ or CBD-AID) was approximately 9-fold more potent than the AID peptide in inhibiting CN phosphatase activity. Similar results were also obtained in the trans-inhibition study of the mouse $\mathrm{CN} \alpha_{1-388}$ (equivalent to human $\mathrm{CN} \beta_{1-397}$ ) (Figure 3E). These results suggest that one or more additional regions might coordinate with AID to mediate the interaction of $\mathrm{RD}_{398-500}$ fragment with $\mathrm{CN}_{1-397}$ [15]. Alternatively, they may also imply that AID and CBD contribute cooperatively to the tight binding of this fragment to $\mathrm{CN}_{1-397}$. Generally, bipartite binding enhances the affinity of individually weaker interactions and allows for greater binding specificity.

CaM-RD complex can directly interact with $C N_{1-397}$, but does not completely abolish its phosphatase activity

The C-terminal truncation assays shown in Figure 3 revealed that CaM-stimulated phosphatase activity of WT CN towards $p$ RII was only about one fourth of those observed for the C-terminal truncation mutants (M1, M2 and M3), and it can be further increased to a fully active level by limited trypsin digestion activation (Supplementary information, Figure S4B), indicating that AID-mediated cis-autoinhibition may not be completely relieved upon CaM binding. It is likely that AID can bind to a region near the active site even when CaM is bound. Given that the isolated regulatory fragment $\left(\mathrm{RD}_{398-500}\right)$ was able to interact with $\mathrm{CaM}$ and the $\mathrm{CN}$ catalytic core $\left(\mathrm{CN}_{1-397}\right)$ individually, we next determined whether binding of CaM to $\mathrm{RD}_{398-500}$ disrupts the interaction of its AID with the catalytic site. We took two approaches to study the effect of CaM binding on the interaction between the disjoint segments, $\mathrm{CN}_{1-397}$ and $\mathrm{RD}_{398-500}$. As shown in Figure $4 \mathrm{~A}, \sim 95 \%$ of the $\mathrm{CN}_{1-397}$ phosphatase activity towards $p$ RII was inhibited by $8 \mu \mathrm{M} \mathrm{RD}_{398-500}$, and this 
A



D $\mathrm{hCN} \beta_{1-397}$ towards $p \mathrm{RII}$

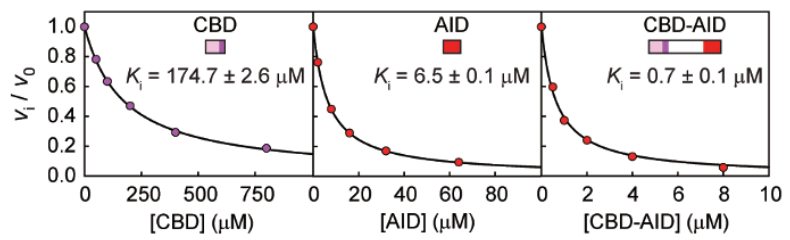

E

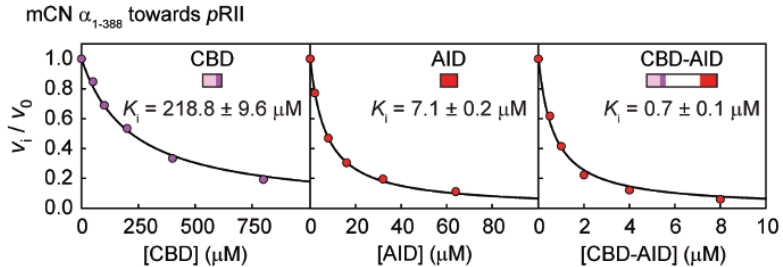

hCN $\beta$ towards $p$ NPP

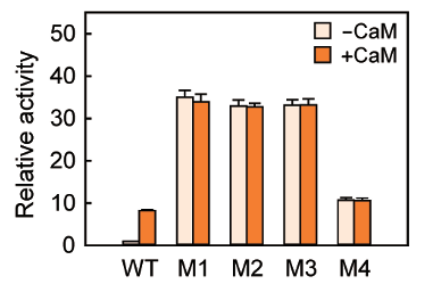

C

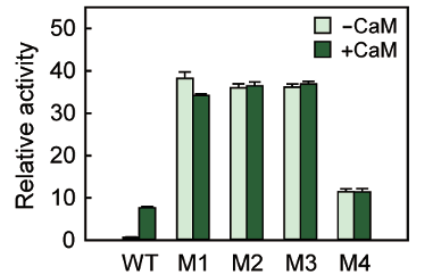

hCN $\beta$ towards $p$ RII



$\mathrm{mCN} \alpha$ towards $p \mathrm{RII}$



Figure 3 Phosphatase activities of WT CN and its truncated forms. (A) Schematic diagram of CNA truncation mutants. (B, C) Activities of WT or mutant human $\mathrm{CN} \beta(\mathbf{B})$ and mouse $\mathrm{CN} \alpha(\mathbf{C})$ towards $p N P P$ or $p$ RIl peptide in the absence or presence of $200 \mathrm{nM}$ CaM (Mean \pm SEM; $n=3$ ). (D, E) Inhibition of the phosphatase activities of human CN $\beta_{1-397}(\mathrm{D})$ and mouse $\mathrm{CN} \alpha_{1-388}(\mathrm{E})$ towards $p$ RII by different autoinhibitory segments. The solid lines represent the best fit of data to the equation $v / v_{0}=K_{i} /\left(K_{i}+[I)\right.$, where $K_{i}$ is the apparent inhibition constant. All experiments have been repeated at least three times. Assays in B-E were performed in the presence of $1 \mathrm{mM} \mathrm{Ca}^{2+}$ and $1 \mathrm{mM} \mathrm{Mn}^{2+}$.

inhibition could only be partially reversed by addition of excess CaM. Consistently, the $\mathrm{CN}_{1-397}$ phosphatase activity can still be partially inhibited (up to $\sim 70 \%$ ) by $\mathrm{RD}_{398-}$ ${ }_{500}$ when an excess of $\mathrm{CaM}$ is present (Figure 4B). These results indicate that the $\mathrm{CaM} / \mathrm{RD}_{398-500}$ complex can still bind to $\mathrm{CN}_{1-397}$, and binding of $\mathrm{CaM}$ to $\mathrm{RD}_{398-500}$ alters its interaction mode with the active site of $\mathrm{CN}_{1-397}$, resulting in an incomplete trans-inhibition. The incomplete inhibition plateauing at $\sim 30 \%$ of the maximal phosphatase activity implies noncompetitive inhibition of $\mathrm{CN}_{1-397}$ by the $\mathrm{CaM} / \mathrm{RD}_{398-500}$ complex. Interestingly, the $\mathrm{CN} / \mathrm{CaM}$ binary complex and the $\mathrm{CN}_{1-397} / \mathrm{CaM} / \mathrm{RD}_{398-500}$ ternary complex displayed similar phosphatase activities towards $p$ RII, suggesting that the cis- and trans-inhibition of CN activity by $\mathrm{CaM} / \mathrm{RD}_{398-500}$ display a similar noncompetitive mode. Similar results were obtained using $p$ NPP as the substrate: the $\mathrm{RD}_{398-500}$ peptide can inhibit $\mathrm{CN}_{1-397}$ activity almost completely in the absence of $\mathrm{CaM}$, while the $\mathrm{CaM} / \mathrm{RD}_{398-500}$ complex resulted in partial inhibition. In addition, $\mathrm{RD}_{398-500}$-induced inhibition can be partially reversed by CaM (Figure 4C).

To further examine whether $\mathrm{CN}_{1-397}, \mathrm{CaM}$ and $\mathrm{RD}_{398-500}$ form a ternary complex, we investigated the interaction between $\mathrm{CN}_{1-397}$ and the $\mathrm{CaM} / \mathrm{RD}_{398-500}$ binary complex through pull-down assay (Figure $4 \mathrm{D}$ ). The $\mathrm{RD}_{398-500}$ fragment bound with GST-tagged CaM efficiently and formed a 1:1 complex on column. This preformed GST$\mathrm{CaM} / \mathrm{RD}_{398-500}$ binary complex was able to pull down the $\mathrm{CN}_{1-397}$ protein while $\mathrm{CaM}$ alone cannot, indicating that $\mathrm{CaM} / \mathrm{RD}_{398-500}$ directly interacts with $\mathrm{CN}_{1-397}$ to form a ternary complex on column. Together, both the trans-inhibition assay and the pull-down assay results indicate that binding of $\mathrm{RD}_{398-500}$ to $\mathrm{CN}_{1-397}$ is not abolished when CaM binds to the CBD in $\mathrm{RD}_{398-500}$. This result appears to be in contradiction to the fluorescence anisotropy experiments reported by Rumi-Masante et al. [25]. They found that although fl- $\mathrm{RD}_{373-521}$, a fluorescently labeled construct, can bind to the truncated $\mathrm{CN}_{1-373}$ (equivalent to $\mathrm{RD}_{382-524}$ and $\mathrm{CN}_{1-382}$ in human $\mathrm{CN} \beta$, respectively) as indicated by an increase in anisotropy, there was no measurable anisotropy change for $\mathrm{fl}-\mathrm{RD}_{373-521}$ in the presence of $\mathrm{CaM}$ or of CaM plus $\mathrm{CN}_{1-373}$. In general, the fluorescence anisotropy can reflect changes in molecular size and shape, and thus it usually increases when the labeled molecules form complexes with other molecules. However, in some cases it may not be possible to obtain a measurable change in fluorescence anisotropy even when the complex is formed. Thus, the absence of a measurable anisotropy change should not be interpreted as an unambiguous demonstration of the absence of interaction [26, 
A hCN $\beta_{1-397}+$ CBD-AID towards $p$ RII

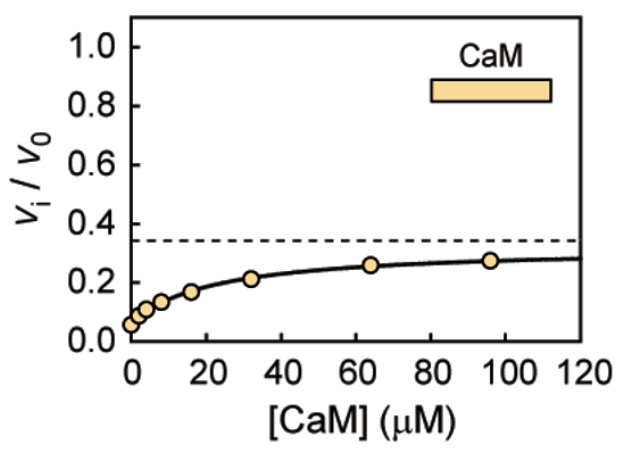

$\mathrm{B}$ hCN $\beta_{1-397}+$ CaM towards $p$ RII

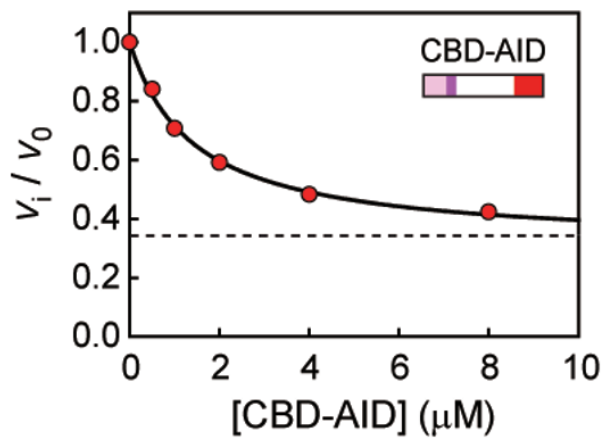

C pNPP as substrate
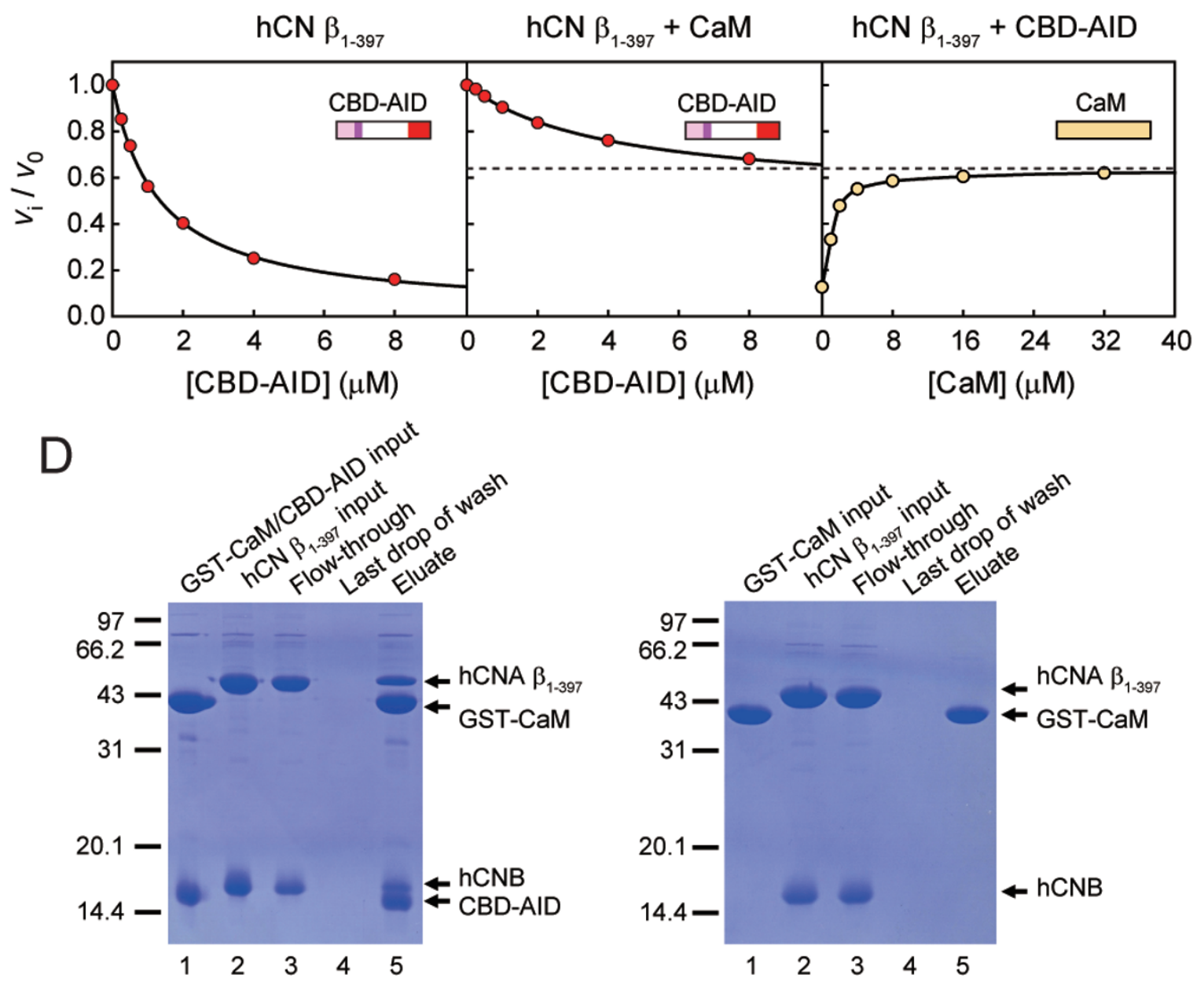

Figure $4 \mathrm{CaM} / \mathrm{RD}_{398-500}$ complex binds to the catalytic core of $\mathrm{CN}$ and inhibits its activity noncompetitively. (A) Effect of CaM on the inhibition of $\mathrm{CN}$ activity by the CBD-AID fragment $\left(\mathrm{RD}_{398-500}\right) \cdot \mathrm{CN}_{1-397}(8 \mathrm{nM})$ was assayed, using $6 \mu \mathrm{M} p \mathrm{RII}$ peptide and

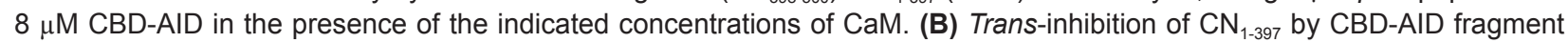
in the presence of excess CaM $(64 \mu \mathrm{M}) .8 \mathrm{nM} \mathrm{CN}_{1-397}$ and $6 \mu \mathrm{M}$ pRII peptide were used. (C) Effect of CBD-AID fragment on the activity of $\mathrm{CN}_{1-397}$ towards $p$ NPP. Assays were performed similarly with that using $p$ RII peptide as the substrate. All experiments have been repeated at least three times. Assays in A-C were performed in the presence of $1 \mathrm{mM} \mathrm{Ca}^{2+}$ and $1 \mathrm{mM} \mathrm{Mn}^{2+}$. (D) Interaction of CaM/CBD-AID with $\mathrm{CN}_{1-397}$ in the presence of $2 \mathrm{mM} \mathrm{Ca}^{2+}$ and $1 \mathrm{mM} \mathrm{Mn}^{2+}$. GST-CaM/CBD-AID (left) or GSTCaM (right) was bound to the glutathione-Sepharose $4 \mathrm{~B}$ resin (lane 1). $\mathrm{CN}_{1-397}$ was allowed to flow through the resin (lanes 2 and 3). After extensive washing (lane 4), the bound proteins were eluted with reduced glutathione (lane 5). Samples were visualized by SDS-PAGE with Coomassie Blue staining. 
27]. Thus, an alternative explanation for the absence of a detectable anisotropy change could be that the conformational change of $\mathrm{RD}_{373-521}$ upon $\mathrm{CaM}$ binding affects the labeled end and the fluorophore in the $\mathrm{RD}_{373-521} / \mathrm{CaM}$ complex might be unable to reflect the protein-protein interaction.

\section{Further evidence for the multi-level activation of $C N$}

$\mathrm{CN}$ has been known as a common target for the immunosuppressant-immunophilin complexes CyPA-CsA and FKBP12-FK506 [7]. A long-standing paradox concerning the regulation of $\mathrm{CN}$ is that the $\mathrm{CN}$ phosphatase activity towards $p$ RII substrate was strongly inhibited by both CyPA-CsA and FKBP12-FK506, while the activity of $\mathrm{CN}$ towards $p$ NPP was stimulated by approximately 4-fold in the presence of the drug complexes [28, 29]. We confirmed these results for WT CN $\beta$. As shown in Figure 5A, in the presence of excess $(200 \mathrm{nM}) \mathrm{CaM}$, the activity of $\mathrm{CN}$ towards $p$ NPP can be further stimulated by addition of the FKBP12-FK506 complex. Activation of $\mathrm{CN}$ was dose-dependent and saturable with the half-maximal effect detected in the presence of $100 \mathrm{nM}$ FKBP12-FK506 complex. Curiously, the phosphatase activity of $\mathrm{CN}_{1-450}$ towards $p$ NPP was not stimulated by the FKBP12-FK506 complex. Effects of the FKBP12-FK506 complex on the activities of $\mathrm{CN}$ and its $\mathrm{C}$-terminally truncated mutants towards $p$ NPP in the presence of CaM are summarized in Figure 5B and 5C. Unlike the native enzyme, all the AID-deleted CN mutants have full enzyme activities which cannot be further increased by the FKBP12-FK506 complex. In accordance with this, the in vitro proteolysis study also showed that the activity of the fully proteolyzed $\mathrm{CN}$ reached its maximum and was not affected by the FKBP12-FK506 complex (Supplementary information, Figure S5). In the crystal structure of CNFKBP12-FK506, the immunosuppressant-immunophilin complex interacts with the same hydrophobic pocket that is recognized by the LxVP motif, and AID is displaced from the active site [9]. A structural superposition of $\mathrm{CN}$ over the CN-FKBP12-FK506 complex showed that FKBP12 interacts and overlaps with AID, but it does not seem to block access to the active site (Figure 5D). Thus, a very likely explanation for the apparent paradox is that AID can still interact with a region near the active site even when CaM binds to $\mathrm{CBD}$ of $\mathrm{CN}$, and binding of the FKBP12-FK506 complex to the CaM-CN complex leads to complete release of AID from the catalytic core.

Direct interactions between AID and the active site in CNA seen in crystal structures suggest that exogenous AID peptide might be a competitive inhibitor of the $\mathrm{CN}$ catalytic domain. However, previous studies have reported conflicting data regarding the mechanism of inhibition with the AID peptide. Non-competitive inhibition has been reported by Perrino and colleagues using $\left[\mathrm{P}^{32}\right] \mathrm{MLC}$ and $\left[\mathrm{P}^{32}\right] \mathrm{RII}$ peptides as substrates $[30,31]$, while competitive inhibition has been suggested by Parsons et al. using the $\left[\mathrm{P}^{32}\right] \mathrm{RII}$ substrate [32]. To reexamine the mechanism of inhibition by the AID peptide, we carried out trans-inhibition experiments using the AID and $p$ RII peptides. In the presence of CaM, the phosphatase activity of WT CN was only partially inhibited by the AID peptide (Figure 5E), whereas the activity of the AID-deleted form $\mathrm{CN}_{1-450}$ can be completely suppressed (Figure $5 \mathrm{~F}$ ). One possible explanation for the incomplete inhibition is that $p$ RII and AID peptides can bind simultaneously to CaM-bound $\mathrm{CN}$ and thus the isolated AID peptide does not completely block the catalytic center. Interestingly, the extent of the inhibitory effect is similar to the inhibition of $\mathrm{CN}_{1-397}$ by $\mathrm{CaM} / \mathrm{RD}_{398-500}$ (Figures $4 \mathrm{~B}$ and $5 \mathrm{E}$ ). The differential trans-inhibition patterns shown in Figure $5 \mathrm{E}$ and $5 \mathrm{~F}$ provide further support for the notion that binding of $\mathrm{CaM}$ to the regulatory region of $\mathrm{CN}$ does not remove AID from the active site, but only regulates the orientation of AID with respect to the catalytic core. The reoriented-AID locates near the active site and restricts free access of the isolated AID peptide. Our results are similar to those obtained by Perrino and colleagues but differ from those of Parsons et al. To further clarify this issue, we also reanalyzed the data shown in Figure 6 of the work by Parsons et al. [32], and found that the inhibitor concentrations used in their experiments were too low to distinguish the binding mode for AID inhibition (Supplementary information, Figure S6).

\section{Molecular mechanisms for $\mathrm{CN}$ regulation}

Based on results presented in this study and the previous knowledge on $\mathrm{CN}$ activation, we propose a modified model for the regulation of CN (Figure 6). In this model, $\mathrm{CN}$ activation involves multiple steps: (1) at low $\mathrm{Ca}^{2+}$ concentration, $\mathrm{CN}$ exists in an inactive state in which only two high-affinity binding sites on CNB are occupied by $\mathrm{Ca}^{2+}$ and several distinct parts of the CNA regulatory region interact with the catalytic core (the catalytic core include the CNA catalytic domain plus $\mathrm{BBH}$ and CNB) (Form I or the resting conformation) [13, 31]; (2) in response to elevated calcium level, occupancy of the low-affinity sites on CNB by $\mathrm{Ca}^{2+}$ induces a conformational change in $\mathrm{CNB}$ and triggers movement of a larger part of the CNA regulatory region, stimulating the basal activity of $\mathrm{CN}$ (Form II, the crystal structures of $\mathrm{CN} \alpha$ and $\beta$ might represent snapshots of the ensemble with different conformations) [13,33]; (3) binding of CaM to $\mathrm{CBD}$, causing $\mathrm{CBD}$ to form an $\alpha$-helix, leads to the dissociation of AIS from the LxVP-docking pocket and 


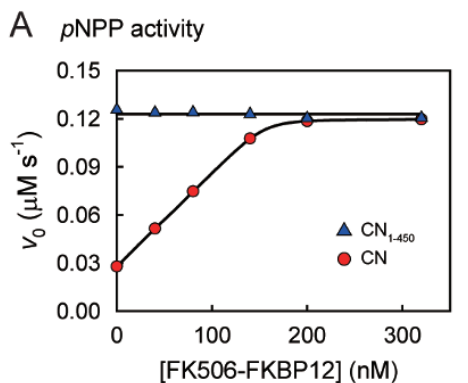

B hCN $\beta$ towards $p N P P$

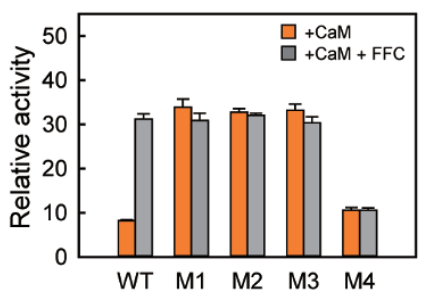

C $\mathrm{mCN} \alpha$ towards $p N P P$



E hCN $\beta$

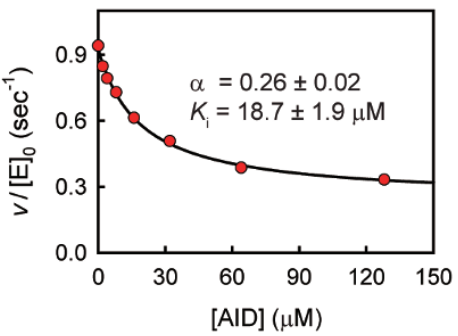

$\mathrm{F} \quad \mathrm{hCN} \beta_{1-450}$



Figure 5 Effects of FKBP12-FK506 complex and AID fragment on the CN activity. (A) Effects of FKBP12-FK506 complex on the $p$ NPP activity of $\mathrm{CN}$ and $\mathrm{CN}_{1-450}$ in the presence of $200 \mathrm{nM} \mathrm{CaM.} \mathrm{(B,} \mathrm{C)} \mathrm{Activities} \mathrm{of} \mathrm{WT} \mathrm{or} \mathrm{mutant} \mathrm{human} \mathrm{CN} \beta$ (B) and mouse $\mathrm{CN} \alpha(\mathbf{C})$ towards $p$ NPP in the presence of CaM (colored) or CaM plus FKBP12-FK506 complexes (FFC, gray) (Mean \pm SEM; $n=3$ ). (D) Structure comparison of CN-FKBP12-FK506 complex structure (PDB ID code: 1TCO) and human CN $\beta$ structure. The CN-FKBP12-FK506 complex is shown in grey and superimposed onto CN $\beta$ which follows the color scheme in Figure 1C. The left and right panels show that FK506 and FKBP12 would sterically clash with AIS (left) and AID (right) of CN, respectively. (E, F) Trans-inhibition of the dephosphorylation activity of $C N \beta(E)$ or $C_{1-450}(\mathbf{F})$ towards $p$ RII by the AID peptide in the presence of $200 \mathrm{nM}$ CaM. The solid lines represent the best fit of data to the equation $v /[E]_{0}=v_{0} /[E]_{0} \times\left(K_{i}+\alpha[I]\right) /\left(K_{i}+[I]\right)$, where $K_{i}$ and $\alpha$ are the apparent inhibition constant and residual activity, respectively. All experiments have been repeated at least three times. All assays were performed in the presence of $1 \mathrm{mM} \mathrm{Ca}^{2+}$ and $1 \mathrm{mM} \mathrm{Mn}^{2+}$.

a conformational rearrangement of the AID segment, which results in further activation of CN (Form III) [25, $34,35]$; (4) $\mathrm{CN}$ is fully activated via a limited hydrolysis which removes the autoinhibitory regions in the C-terminus of CNA (Form IV) [36]. This new model can also be used to explain the truncation experiments and trans-inhibition data shown in Figures 3-5.

In summary, our data probe the mechanism by which $\mathrm{CaM}$ activates the phosphatase $\mathrm{CN}$. However, our new model does not concern the resting conformation of $\mathrm{CN}$ at low $\mathrm{Ca}^{2+}$ concentration. There is some existing evidence suggesting different $\mathrm{CN}$ conformations between the resting and fully $\mathrm{Ca}^{2+}$-loaded states (Form I and Form
II). (1) The entire CNA regulatory region is substantially protected from enzymatic digestion at a sufficiently low concentration of $\mathrm{Ca}^{2+}$, suggesting a structured conformation of the CNA regulatory region in the resting state (Form I), in which CBD and BBH interact with each other [13]. In the absence of $\mathrm{Ca}^{2+}$, kinetic analysis of WT and $\mathrm{C}$-terminally truncated $\mathrm{CN} \alpha$ mutants has provided further evidence for the presence of additional autoinhibitory elements within residues 420-457 (C-terminal to AIS) [31]. (2) A proteolyzed derivative of $\mathrm{CN}, \mathrm{CN}-45$, which no longer binds $\mathrm{CaM}$, is strikingly activated by $\mathrm{Ca}^{2+}$ binding to CNB. Similarly, the $\mathrm{CN}_{420}$ truncation mutant that includes CBD responds to $\mathrm{Ca}^{2+}$ alone (with a de- 

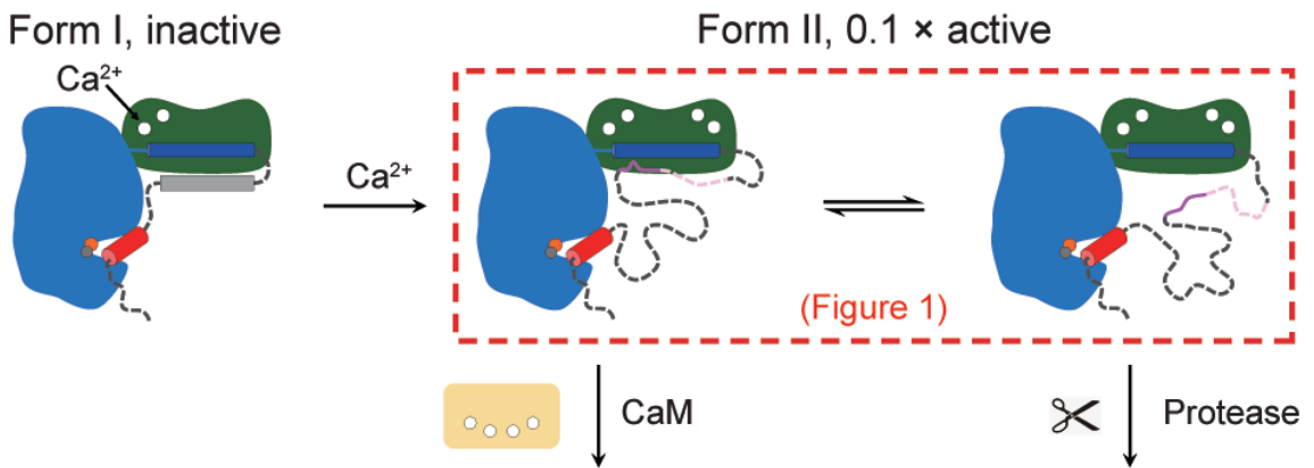

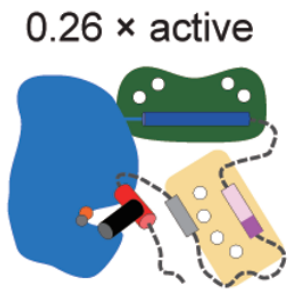

(Figure 5E)

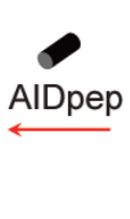

Form III, $1 \times$ active

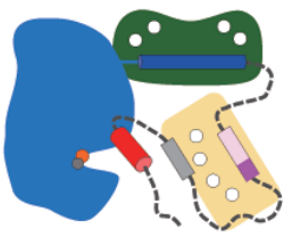

(Figure 3B)

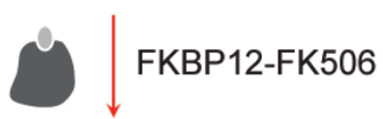

$4 \times$ active for $p N P P$

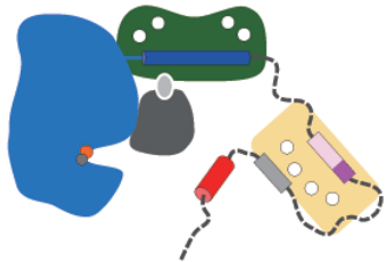

(Figure $5 \mathrm{~A}$ )
Form IV, $4 \times$ active

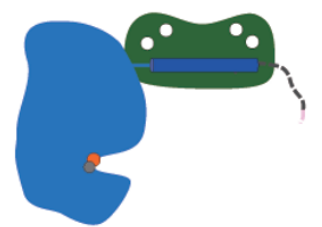

(Figure 3B)

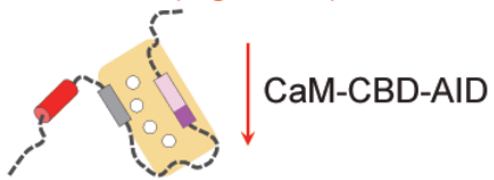

$1 \times$ active



(Figure 4B)

Figure 6 Cartoon model for the $\mathrm{CN}$ regulation. The gray box in Form I represents a structured region in resting CN. A detailed description of the model is given in the text.

creased $K_{\mathrm{m}}$ for $p$ RII peptide). These results suggest that the interaction of $\mathrm{CBD}$ and $\mathrm{BBH}$ in the resting form is regulated by $\mathrm{Ca}^{2+} / \mathrm{B}$ subunit $[13,31,33]$. (3) It has been reported that differences in experimental conditions, such as the use of specific metal ions, $\mathrm{Mn}^{2+}, \mathrm{Mg}^{2+}$ and $\mathrm{Ni}^{2+}$, may produce altered kinetic behavior of the $\mathrm{CN}$ enzyme $[18,31,32]$. Therefore, the conformation of $\mathrm{CN}$ in the presence of $\mathrm{Mn}^{2+}$ might differ from the physiological conformation. The crystal structure of full-length $\mathrm{CN}$ in the resting state will ultimately illustrate the mechanism of $\mathrm{Ca}^{2+}$-dependent $\mathrm{CN}$ activation.

\section{Discussion}

Intracellular calcium is a powerful secondary messen- ger that affects a number of calcium sensors, including $\mathrm{CaM}, \mathrm{Ca}^{2+} / \mathrm{CaM}$-dependent protein kinases, $\mathrm{CN}$, and calpain (a $\mathrm{Ca}^{2+}$-dependent cysteine protease) [37]. In addition to the conventional $\mathrm{Ca}^{2+} / \mathrm{CaM}$-triggered activation pathway, $\mathrm{CN}$ can also be directly activated by calpain through proteolysis in vitro and in vivo [38]. Three calpain-dependent truncated forms of CNA have been identified, i.e., CNA fragments 1-392 (45 kDa, similar to Form IV in the CN activation model shown in Figure 6), 1-424 (48 kDa, an AID-deleted form of CN) and 1-501 (57 $\mathrm{kDa}$ ). Proteolysis removes AID (in the 45 and $48 \mathrm{kDa}$ forms) and changes the phosphatase to its constitutively active form. In cultured hippocampal neurons, overexpressing an adenoviral-based $48 \mathrm{kDa}$ CNA truncated form induces caspase activation and neuronal cell death. 
Moreover, calpain activation and the production of 45-48 $\mathrm{kDa}$ truncation of CNA are associated with glutamateand kainate-induced excitotoxicity in hippocampal neurons [38]. Thus, overactivation of the calpain-CN pathway could contribute to calcium-dependent disorders, especially those associated with Alzheimer's disease and myocardial hypertrophy $[39,40]$.

$\mathrm{CaM}$ is a ubiquitous protein that plays a key role in calcium-mediated signal transduction. Among numerous serine/threonine protein kinases, there is a distinct subfamily of $\mathrm{Ca}^{2+} / \mathrm{CaM}$-dependent kinases, all of which contain a CBD and an AID [41]. The CaM-binding site is immediately adjacent in sequence to AID, and activation of these kinases requires the removal of AID from the active site. In contrast to CaM-activated kinases, $\mathrm{CN}$ is the only one CaM-activated phosphatase known. Since the AID in $\mathrm{CN}$ is located $\sim 50$ residues C-terminal of the CBD, and most of the C-terminal region of CNA apart from AID (457-482) is missing from previously published structures, how the binding of CaM to CBD of $\mathrm{CN}$ transmits through the $\sim 50$-residue linker to displace AID from the catalytic site is a topic of ongoing debate. Recently, Rumi-Masante and colleagues used CD spectroscopy, hydrogen-deuterium exchange mass spectrometry, and limited proteolytic digestions to show that the isolated RD fragment of $\mathrm{CN}$ is disordered but gains structure upon CaM binding [25, 34]. This structure includes the expected $\alpha$-helix in CBD and a so-called distal helix lying somewhere between the end of CBD and the beginning of AID. The distal helix may fold onto the remainder of the $\mathrm{CaM} / \mathrm{RD}$ complex, altering the conformation of the ordered segment of AID. Similarly, Zhao et al. [35] determined the overall secondary structure of $\mathrm{CN}$ in the absence or presence of $\mathrm{Ca}^{2+} / \mathrm{CaM}$ using Fourier transform infrared spectroscopy (FT-IR), and found that the $\mathrm{RD}$ of $\mathrm{CN}$ undergoes $\mathrm{CaM}$-induced large-scale folding, transforming into a more constrained conformation. These results revealed that the binding of $\mathrm{Ca}^{2+}$ and subsequent binding of $\mathrm{CaM}$ generated sequential folding of $\mathrm{CN}$, transforming it into a more constrained, less flexible conformation, suggesting that the intervening disordered residues play important roles in the regulation of $\mathrm{CN}$ activity both in the absence and presence of CaM.

One of the two major claims made in this study is that $\mathrm{CaM}$ binding does not remove AID from the active site. The first evidence for this comes from our phosphatase activity measurements made on full-length $\mathrm{CN}$ and various truncation mutants (Figure 3). The CaM-bound full-length $\mathrm{CN}$ has lower activity than the truncation mutants lacking AID and can be converted into a fully active state by limited trypsin digestion. This finding does not agree with some previously published data for the activity of similarly truncated $\mathrm{CN}$ molecules. The pioneering work of Klee and colleagues [12, 13, 33, 36] sketched out the steps in activation of $\mathrm{CN}$, yet a concrete structural information of these steps is still elusive. For example, Hubbard and Klee reported that the activity of clostripain-proteolyzed 43-kDa CN $\alpha$ (which contains the CNB-binding domain) is about 4-fold higher than that of the WT enzyme in the presence of $\mathrm{Ca}^{2+} / \mathrm{CaM}$. Similar results were also obtained by Wang et al. using the truncated form of $\mathrm{CN} \alpha$ [14]. In contrast, Shen et al. found that the activity of similar truncated $\mathrm{CN} \alpha$ was the same as that of the native enzyme [42]. To address this issue, we reexamined the expression and phosphatase activity of similarly truncated $\mathrm{CN} \beta$ forms generated by conventional methods (Supplementary information, Figure S7). Most $\mathrm{CN}$ mutations $\left(\mathrm{CN}_{1-475}, \mathrm{CN}_{1-429}\right.$ and $\mathrm{CN}_{1}$. ${ }_{414}$ ) dramatically reduced the solubility of the truncated proteins when expressed in E. coli. These mutants partitioned partly into inclusion bodies, which might reflect the aggregation and accumulation of improperly folding intermediates. As shown in Supplementary information, Figure $\mathrm{S} 7$, these $\mathrm{CN}$ truncation mutants (including $\mathrm{CN}_{1}$. ${ }_{414}$ ) showed only approximately 2 -fold higher activity than that of the CaM-stimulated WT CN. Thus, one possible explanation for these discrepancies might be that the soluble protein samples may also contain some misfolding molecules, giving rise to the conflicting results when measuring their activities.

In summary, understanding molecular basis for $\mathrm{CN}$ activation will allow us to better comprehend complex signaling pathways and biological processes. The proposed multi-level regulatory mechanisms provide new insights into how $\mathrm{CN}$ interacts with its binding partners. Although AIS, A238L, $p$ RII peptide and immunosuppressant drugs are functionally distinct, they share a similar mechanism of action with a common docking site in $\mathrm{CN}$, highlighting the importance of such interactions in regulation of the CN/NFAT signaling pathway. This finding may make it possible to develop a structure-based approach for the design of the next-generation $\mathrm{CN}$ inhibitors. Future studies will undoubtedly continue to investigate the complex regulatory mechanisms of $\mathrm{CN}$, such as structural analyses of the resting and CaM-stimulated $\mathrm{CN}$ (Form I and Form III).

\section{Materials and Methods}

\section{Protein purification}

$\mathrm{CaM}$ and the full-length or truncated proteins of $\mathrm{CN}$ were overexpressed in E. coli with His- or GST-tag. The site-specific mutations were generated by overlap PCR procedure. All proteins were purified over affinity, ion-exchange and size-exclusion columns. Protein stocks for phosphatase assays were supplemented with 
glycerol to final concentration of $20 \%(\mathrm{v} / \mathrm{v})$.

\section{Crystallography}

Crystals of WT or mutant $\mathrm{CN} \beta$ were grown by mixing proteins with equal volume of reservoir solutions containing 100 mM MES, pH 5.8 and 7\%-9\% PEG3350. Crystals of CN $\alpha$ were obtained by mixing protein with an equal volume of reservoir solution containing $100 \mathrm{mM}$ MES, pH 6.1, 18\% PEG3350, 8\% Glycerol and $0.2 \mathrm{M} \mathrm{CaCl}_{2}$. The diffraction data sets were collected at beamline BL17U at the Shanghai Synchrotron Radiation Facility and beamline BL41XU at SPring-8. All structures were solved by molecular replacement, and the data processing and refinement statistics were summarized in Supplementary information, Table $\mathrm{S} 1$. The coordinates and structure factors have been deposited in the Protein Data Bank (PDB) with accession codes 4OR9 for the human $\mathrm{CN} \beta$ structure, 4ORA for the $\mathrm{CN}_{\mathrm{V} 418 \mathrm{Y} / \mathrm{F} 419 \mathrm{~L}}$ mutant structure and $4 \mathrm{ORB}$ for the mouse $\mathrm{CN} \alpha$ structure.

\section{CN phosphatase assay}

The phosphatase activity of $\mathrm{CN}$ was assayed using $p$ NPP or $p$ RII peptide as substrate in the presence of $1 \mathrm{mM} \mathrm{Ca}^{2+}$ and $1 \mathrm{mM}$ $\mathrm{Mn}^{2+}$, and the continuous absorbance changes were recorded with a PerkinElmer LAMBDA 45 spectrophotometer. The initial rates were determined from the linear slope of the progress curves. Trans-inhibition studies of CN were performed with increasing concentrations of various inhibitory fragments. The apparent inhibition constants were determined by fitting the experimental data sets to equations for trans-inhibition.

More methods and details were provided in Supplementary information, Data S1.

\section{Acknowledgments}

We thank the staff members of beamlines BL17U at SSRF and BL41XU at SPring-8. This work was supported by the Ministry of Science and Technology of China ( 2013CB530600), and the National Science Foundation of China (31130062, 31270848 and 31321003).

\section{Author Contributions}

SJL, JW, and LM performed research and analyzed the data. CL and JW contributed to data analysis. ZXW and JWW designed research and wrote the paper with input from all authors.

\section{Competing Financial Interests}

The authors declare no competing financial interests.

\section{References}

1 Aramburu J, Rao A, Klee CB. Calcineurin: from structure to function. Curr Top Cell Regul 2000; 36:237-295.

2 Rusnak F, Mertz P. Calcineurin: form and function. Physiol Rev 2000; 80:1483-1521.

3 Li H, Rao A, Hogan PG. Interaction of calcineurin with substrates and targeting proteins. Trends Cell Biol 2011; 21:91103.

4 Crabtree GR, Olson EN. NFAT signaling: choreographing the social lives of cells. Cell 2002; 109:S67-S79.

5 Macian F. NFAT proteins: key regulators of T-cell develop- ment and function. Nat Rev Immunol 2005; 5:472-484.

6 Mancini M, Toker A. NFAT proteins: emerging roles in cancer progression. Nat Rev Cancer 2009; 9:810-820.

7 Liu J, Farmer JD Jr, Lane WS, Friedman J, Weissman I, Schreiber SL. Calcineurin is a common target of cyclophilin-cyclosporin A and FKBP-FK506 complexes. Cell 1991; 66:807815.

8 Griffith JP, Kim JL, Kim EE, et al. X-ray structure of calcineurin inhibited by the immunophilin-immunosuppressant FKBP12-FK506 complex. Cell 1995; 82:507-522.

9 Kissinger CR, Parge HE, Knighton DR, et al. Crystal structures of human calcineurin and the human FKBP12-FK506-calcineurin complex. Nature 1995; 378:641644.

10 Huai Q, Kim HY, Liu Y, et al. Crystal structure of calcineurin-cyclophilin-cyclosporin shows common but distinct recognition of immunophilin-drug complexes. Proc Natl Acad Sci USA 2002; 99:12037-12042.

11 Jin L, Harrison SC. Crystal structure of human calcineurin complexed with cyclosporin A and human cyclophilin. Proc Natl Acad Sci USA 2002; 99:13522-13526.

12 Klee CB, Ren H, Wang X. Regulation of the calmodulin-stimulated protein phosphatase, calcineurin. J Biol Chem 1998; 273:13367-13370.

13 Yang SA, Klee CB. Low affinity $\mathrm{Ca}^{2+}$-binding sites of calcineurin B mediate conformational changes in calcineurin A. Biochemistry 2000; 39:16147-16154.

14 Wang H, Du Y, Xiang B, Lin W, Li X, Wei Q. A renewed model of CNA regulation involving its $\mathrm{C}$-terminal regulatory domain and CaM. Biochemistry 2008; 47:4461-4468.

15 Perrino BA. Regulation of calcineurin phosphatase activity by its autoinhibitory domain. Arch Biochem Biophys 1999; 372:159-165.

16 Ye Q, Feng Y, Yin Y, et al. Structural basis of calcineurin activation by calmodulin. Cell Signal 2013; 25:2661-2667.

17 Kilka S, Erdmann F, Migdoll A, Fischer G, Weiwad M. The proline-rich N-terminal sequence of calcineurin Abeta determines substrate binding. Biochemistry 2009; 48:1900-1910.

18 Sagoo JK, Fruman DA, Wesselborg S, Walsh CT, Bierer BE. Competitive inhibition of calcineurin phosphatase activity by its autoinhibitory domain. Biochem $J$ 1996; 320:879-884.

19 Quintana AR, Wang D, Forbes JE, Waxham MN. Kinetics of calmodulin binding to calcineurin. Biochem Biophys Res Commun 2005; 334:674-680.

20 Li H, Zhang L, Rao A, Harrison SC, Hogan PG. Structure of calcineurin in complex with PVIVIT peptide: portrait of a low-affinity signalling interaction. J Mol Biol 2007; 369:1296-1306.

21 Roy J, Li H, Hogan PG, Cyert MS. A conserved docking site modulates substrate affinity for calcineurin, signaling output, and in vivo function. Mol Cell 2007; 25:889-901.

22 Li H, Pink MD, Murphy JG, Stein A, Dell'Acqua ML, Hogan PG. Balanced interactions of calcineurin with AKAP79 regulate $\mathrm{Ca}^{2+}$-calcineurin-NFAT signaling. Nat Struct Mol Biol 2012; 19:337-345.

23 Rodriguez A, Roy J, Martinez-Martinez S, et al. A conserved docking surface on calcineurin mediates interaction with substrates and immunosuppressants. Mol Cell 2009; 33:616-626.

24 Grigoriu S, Bond R, Cossio P, et al. The molecular mecha- 
nism of substrate engagement and immunosuppressant inhibition of calcineurin. PLoS Biol 2013; 11:e1001492.

25 Rumi-Masante J, Rusinga FI, Lester TE, et al. Structural basis for activation of calcineurin by calmodulin. J Mol Biol 2012; 415:307-317.

26 Heyduk T, Ma Y, Tang H, Ebright RH. Fluorescence anisotropy: rapid, quantitative assay for protein-DNA and protein-protein interaction. Methods Enzymol 1996; 274:492-503.

27 Anderson BJ, Larkin C, Guja K, Schildbach JF. Using fluorophore-labeled oligonucleotides to measure affinities of protein-DNA interactions. Methods Enzymol 2008; 450:253-272.

28 Swanson SK, Born T, Zydowsky LD, et al. Cyclosporin-mediated inhibition of bovine calcineurin by cyclophilins A and B. Proc Natl Acad Sci USA 1992; 89:3741-3745.

29 Yin M, Ochs RS. Mechanism for the paradoxical inhibition and stimulation of calcineurin by the immunosuppresive drug tacrolimus (FK506). Arch Biochem Biophys 2003; 419:207213.

30 Hashimoto Y, Perrino BA, Soderling TR. Identification of an autoinhibitory domain in calcineurin. J Biol Chem 1990; 265:1924-1927.

31 Perrino BA, Ng LY, Soderling TR. Calcium regulation of calcineurin phosphatase activity by its B subunit and calmodulin. Role of the autoinhibitory domain. J Biol Chem 1995; 270:340-346.

32 Parsons JN, Wiederrecht GJ, Salowe S, et al. Regulation of calcineurin phosphatase activity and interaction with the FK506.FK-506 binding protein complex. J Biol Chem 1994; 269:19610-19616.

33 Stemmer PM, Klee CB. Dual calcium ion regulation of calcineurin by calmodulin and calcineurin B. Biochemistry 1994;
33:6859-6866.

34 Dunlap TB, Cook EC, Rumi-Masante J, Arvin HG, Lester TE, Creamer TP. The distal helix in the regulatory domain of calcineurin is important for domain stability and enzyme function. Biochemistry 2013; 52:8643-8651.

35 Zhao Y, Yang H, Meng K, Yu S. Probing the $\mathrm{Ca}^{2+} / \mathrm{CaM}-i n-$ duced secondary structural and conformational changes in calcineurin. Int J Biol Macromol 2014; 64:453-457.

36 Hubbard MJ, Klee CB. Functional domain structure of calcineurin A: mapping by limited proteolysis. Biochemistry 1989; 28:1868-1874.

37 Wu HY, Tomizawa K, Matsui H. Calpain-calcineurin signaling in the pathogenesis of calcium-dependent disorder. Acta Med Okayama 2007; 61:123-137.

38 Wu HY, Tomizawa K, Oda Y, et al. Critical role of calpain-mediated cleavage of calcineurin in excitotoxic neurodegeneration. J Biol Chem 2004; 279:4929-4940.

39 Liu F, Grundke-Iqbal I, Iqbal K, Oda Y, Tomizawa K, Gong CX. Truncation and activation of calcineurin A by calpain I in Alzheimer disease brain. J Biol Chem 2005; 280:3775537762.

40 Burkard N, Becher J, Heindl C, Neyses L, Schuh K, Ritter O. Targeted proteolysis sustains calcineurin activation. Circulation 2005; 111:1045-1053.

41 Swulius MT, Waxham MN. $\mathrm{Ca}^{2+} /$ calmodulin-dependent protein kinases. Cell Mol Life Sci 2008; 65:2637-2657.

42 Shen X, Li H, Ou Y, et al. The secondary structure of calcineurin regulatory region and conformational change induced by calcium/calmodulin binding. J Biol Chem 2008; 283:11407-11413.

(Supplementary information is linked to the online version of the paper on the Cell Research website.) 